PROCEEDINGS OF THE

AMERICAN MATHEMATICAL SOCIETY

Volume 125, Number 11, November 1997, Pages 3257-3258

S 0002-9939(97)03963-4

\title{
HARMONIC POLYNOMIALS AND THE DIVISIBILITY PROBLEM
}

\author{
V. V. KARACHIK
}

(Communicated by J. Marshall Ash)

\begin{abstract}
An easy way to construct a fist harmonic polynomial component of any polynomial is given.
\end{abstract}

If we tried to divide any polynomial $P(x)\left(x \in R^{n}\right)$ by the polynomial $L(x)$ using the ordinary Euclidean algorithm then for $n>1$ we would meet the problem: What should be a residue? We would not find a reasonable answer if we were keeping in mind that the degree of a residue must be less than the degree of $L(x)$. Nevertheless we may define a "division" of the polynomial $P(x)$ by the polynomial $L(x)$ by the equality $P(x)=Q(x) L(x)+H(x)$, where the residue $H(x)$ is determined not as a polynomial of degree less than degree of $L(x)$ but as a polynomial solution of the equation $L(D) u(x)=0$. Here operator $L(D)$ is obtained from the polynomial $L(x)$ by replacing each variable $x_{i}$ on the differential operator $\partial / \partial x_{i}$. In this case for each polynomial $P(x)$ there exist the only polynomials $Q(x)$ and $H(x)$ such that the equality $P(x)=Q(x) L(x)+H(x)$ holds under the condition $L(D) H(x)=0[1]$. If $L(x)=|x|^{2} \equiv x_{1}^{2}+\cdots+x_{n}^{2}$ this fact was proved in [2]. The proof's method of the above statement does not permit us to construct the polynomial $H(x)$ by the polynomial $P(x)$. In the general case it is not a simple problem. Let us consider an easy way to find the polynomial $H(x)$ for the special form of the polynomial $L(x)$, i.e., if $L(x)=|x|^{2}$ and $n>2$.

Let $L(D)$ be the Laplace operator, i.e., $L(D)=\Delta$.

Lemma. Let $H_{m}(x)$ be a homogeneous harmonic polynomial of $m$-th degree, $H_{m}^{*}(x)$ be the Kelvin transformation of $H_{m}(x)\left(H_{m}^{*}(x) \equiv|x|^{2-n} H_{m}\left(x /|x|^{2}\right)\right)$ and $(k, 2)_{m}=$ $k(k+2) \ldots(k+2 m-2)$. If $n>2$ and $x \neq 0$ then the following formula holds:

$$
H_{m}^{*}(x)=\frac{(-1)^{m}}{(n-2,2)_{m}} H_{m}(D)|x|^{2-n} .
$$

Proof. We shall employ the induction on $m$. Set $m=1$. Then for $k=1, \ldots, n$ we can easily write the equality

$$
\frac{\partial}{\partial x_{k}} \frac{|x|^{2-n}}{2-n}=\frac{1}{|x|^{n-2}} \frac{x_{k}}{|x|^{2}} \equiv x_{k}^{*},
$$

and therefore for $m=1$ Eq.(1) is true.

Received by the editors June 9, 1995 and, in revised form, May 9, 1996.

1991 Mathematics Subject Classification. Primary 31A05; Secondary 11C08.

Key words and phrases. Harmonic polynomials, divisibility.

(C) 1997 American Mathematical Society 
Suppose that for $m<k$ the lemma is true and prove it for $m=k$. Let us use the Euler formula for homogeneous functions. We get

$$
H_{k}(x)=\sum_{i=1}^{n} \frac{x_{i}}{k} H_{k}^{(i)}(x)
$$

where $H_{k}^{(i)}(x)=\partial / \partial x_{i} H_{k}(x)$. Obviously the polynomials $H_{k}^{(i)}(x)$ are harmonic polynomials of degree $k-1$. Making use of Eq.(2), by the induction hypotheses we can write

$$
\begin{aligned}
& H_{k}(D)|x|^{2-n}=\sum_{i=1}^{n} \frac{1}{k} \frac{\partial}{\partial x_{i}} H_{k}^{(i)}(D)|x|^{2-n} \\
= & \sum_{i=1}^{n} \frac{(-1)^{k-1}}{k}(n-2,2)_{k-1} \frac{\partial}{\partial x_{i}}\left(H_{k}^{(i)}\right)^{*}(x) .
\end{aligned}
$$

Keeping in mind that

$$
\sum_{i=1}^{n} \frac{\partial}{\partial x_{i}}\left(H_{k}^{(i)}\right)^{*}(x)=-(2 k+n-4) \sum_{i=1}^{n}\left(x_{i} H_{k}^{(i)}\right)^{*}(x),
$$

we get

$$
H_{k}(D)|x|^{2-n}=(-1)^{k}(n-2,2)_{k-1}(2 k+n-4)\left(\sum_{i=1}^{n} \frac{x_{i}}{k} H_{k}^{(i)}\right)^{*}(x) .
$$

Again, making use of Eq.(2) and observing that $(n-2,2)_{k-1}(2 k+n-4)=$ $(n-2,2)_{k}$ we get Eq.(1), and the proof is complete.

Let $P(x)$ be an arbitrary polynomial. Represent it in the form $P(x)=\sum_{m} P_{m}(x)$.

Theorem. Suppose that $P(x)=Q(x)|x|^{2}+H(x)$ and $H(x)$ is a harmonic polynomial. Then $H(x)$ can be found from the equality

$$
H(x)=\sum_{m}(-1)^{m} \frac{|x|^{2 m+n-2}}{(n-2,2)_{m}} P_{m}(D)|x|^{2-n} .
$$

Proof. Since $P(x)=Q(x)|x|^{2}+H(x)$, then for $x \neq 0$ we get

$$
P_{m}(D)|x|^{2-n}=H_{m}(D)|x|^{2-n} .
$$

If we take advantage of the lemma then we easily get the desired result.

\section{REFERENCES}

[1] V. V. Karachik, O polinomialnyh reshenijah sistem linejnyh differenzialnyh uravnenij. Voprosi Vychislitelnoy i prikladnoy matematiki. v.82, Tashkent, 1987, s.41-48. MR 91f:34008

[2] E. M. Stein, G. Weiss, Introduction to Fourier Analysis on Euclidean Spaces, Princeton, New Jersey, Princeton University Press, 1971. MR 46:4102

Institute of Cybernetics of the Academy of Science of Uzbekistan, 34, F. Hodzhaev St., Tashkent, 700143, Uzbekistan

E-mail address: karachik@uwed.freenet.uz 